\section{Impaired nodal shrinkage and apoptosis define the independent adverse outcome of NOTCH1 mutated patients under ibrutinib therapy in chronic lymphocytic leukemia}

\author{
Giovanni Del Poeta, ${ }^{1}$ Annalisa Biagi, ${ }^{1}$ Luca Laurenti, ${ }^{2}$ Annalisa Chiarenza, ${ }^{3}$ \\ Federico Pozzo, ${ }^{4}$ Idanna Innocenti, ${ }^{2}$ Massimiliano Postorino, ${ }^{1}$ \\ Francesca Maria Rossi, ${ }^{4}$ Maria Ilaria Del Principe, ${ }^{1}$ Riccardo Bomben, ${ }^{4}$ \\ Paolo de Fabritiis, ${ }^{1}$ Antonio Bruno, ${ }^{1}$ Maria Cantonetti, ${ }^{1}$ \\ Francesco Di Raimondo, ${ }^{3}$ Antonella Zucchetto ${ }^{4}$ and Valter Gattei ${ }^{4}$
}

${ }^{1}$ Division of Hematology, Department of Biomedicine and Prevention, University Tor Vergata, Roma; ${ }^{2}$ Division of Hematology, Università Cattolica del Sacro Cuore, Roma; ${ }^{3}$ Division of Hematology, Ferrarotto Hospital, Catania and ${ }^{4} \mathrm{Clinical}$ and Experimental Hematology Unit, Centro di Riferimento Oncologico di Aviano (CRO), IRCCS, Aviano (PN), Italy.

\section{ABSTRACT}

T he introduction of agents inhibiting the B-cell receptor-associated kinases such as ibrutinib has dramatically changed treatments algorithms of chronic lymphocytic leukemia (CLL) as well as the role of different adverse prognosticators. We evaluated the efficacy of ibrutinib as a single agent, in a real-life context, in 180 patients with CLL mostly pretreated, recruited from three independent cohorts from Italy. Patients received $420 \mathrm{mg}$ oral ibrutinib once daily until progression or occurrence of unacceptable side effects. Seventy-three patients discontinued ibrutinib for progression or for adverse events. NOTCH1 mutations (NOTCH1 M) were correlated with a reduced redistribution lymphocytosis, calculated at 3 months on ibrutinib $(P=0.022)$. Moreover, NOTCH1 $M$ patients showed inferior nodal response at 6 months on ibrutinib compared to NOTCH1 wild-type patients $(P<0.0001)$. Significant shorter progression free survival (PFS) and overall survival (OS) were observed in NOTCH1 $M$ patients $(P=0.00002$ and $P=0.001)$. Interestingly, NOTCH1 $M$ plus a lower BAX/BCL-2 ratio identified a CLL subset showing the worst PFS and OS $(P=0.0002$ and $P=0.005)$. In multivariate analysis of PFS and OS, NOTCH1 $M$ were confirmed an independent prognosticator $(P=0.00006$ and $P=0.0039$ ). In conclusion, NOTCH1 $M$ are strongly associated with a lower BAX/BCL-2 ratio, consistent with defective apoptosis, lower redistribution lymphocytosis and lower nodal shrinkage under ibrutinib treatment, this last paramter being responsible for partial responses, subsequent relapses, as well as shorter PFS and OS. Either new small molecule combination approaches or antibodies targeting NOTCH1 could be future therapeutic options for NOTCH1 M patients.

\section{Introduction}

Chronic lymphocytic leukemia (CLL) is the most frequent adult leukemia in Western countries and it is characterized by an extremely heterogeneous clinical course. New molecular aberrations with negative prognostic value in CLL, such as NOTCH1, MYD88, TP53 and SF3B1 gene mutations, were identified in the last decade mainly thanks to the advent of next-generation sequencing (NGS) ${ }^{1,2}$ In particular NOTCH1 mutations $(M)$ are found in $10-14 \%$ of patients at diagnosis with frequency increasing with disease progression and during transformation to Richter syndrome. ${ }^{3}$ Furthermore NOTCH1 $M$ are associated with the presence of trisomy 12 and with high CD49d expression which are negative prognostic factors in CLL. NOTCH1 $M$ are also associated with an increased activation of the NF-kB pathway, promoting tumour cell proliferation and survival. ${ }^{4} \mathrm{NOTCH} 1 \mathrm{M}$ were shown to affect
Ferrata Storti Foundation

Haematologica 2021

Volume 106(9):2345-2353

Correspondence:

GIOVANNI DEL POETA

g.delpoeta@tin.it

Received: February 29, 2020.

Accepted: July 20, 2020.

Pre-published: July 30, 2020.

https://doi.org/10.3324/haematol.2020.251488

(C)2021 Ferrata Storti Foundation

Material published in Haematologica is covered by copyright. All rights are reserved to the Ferrata Storti Foundation. Use of published material is allowed under the following terms and conditions:

https://creativecommons.org/licenses/by-nc/4.0/legalcode. Copies of published material are allowed for personal or internal use. Sharing published material for non-commercial purposes is subject to the following conditions:

https://creativecommons.org/licenses/by-nc/4.0/legalcode, sect. 3. Reproducing and sharing published material for commercial purposes is not allowed without permission in writing from the publisher. 
the response to chemo-immunotherapy in CLL. In the CLL8 study Stilgenbauer et al..$^{5}$ demonstrated that patients carrying NOTCH1 $M$ did not benefit of the addition of rituximab to standard fludarabine and cyclophosphamide chemotherapy. Moreover, it has emerged that NOTCH1 M are associated with decreased duration of response in a large series of relapsed/refractory $(\mathrm{R} / \mathrm{R})$ patients treated with venetoclax. ${ }^{6}$

In a recent study, Tissino et al. ${ }^{7}$ have demonstrated that patients with CLL whose cells were characterized by high CD49d expression, underwent reduced lymphocytosis and inferior nodal response after treatment with ibrutinib. Several reports confirmed that in CLL the balance between the pro- and anti-apoptotic members of the BCL-2 family determines chemotherapy sensitivity and cell survival.,9 Noteworthy, we demonstrated that a low BAX/BCL-2 ratio had an additive negative prognostic impact in both TP53 M and NOTCH1 M patients with CLL treated with chemoimmunotherapy. ${ }^{10}$ The recent introduction of novel B-cell receptor inhibitors such as ibrutinib and idelalisib and of novel potent oral $\mathrm{BH} 3$ peptidomimetics such as venetoclax in clinical practice, prompted us to evaluate the clinical impact of both NOTCH $M$ and BAX/BCL-2 ratio in patients treated with targeted oral therapies and in particular in those treated with ibrutinib.

The aims of this study were: i) to analyse the correlations between NOTCH1 $M$ and other biological parameters including CD49d expression and the BAX/BCL-2 ratio; ii) to address the impact of NOTCH1 $M$ both on redistribution lymphocytosis and on nodal responses after treatment with ibrutinib; iii) to evaluate the impact of NOTCH1 $M$ and $\mathrm{BAX} / \mathrm{BCL}-2$ ratio on the overall response rate (ORR) to ibrutinib, progression free survival (PFS) and overall survival (OS); iiii) to assess whether NOTCH1 $M$ could be considered an independent prognostic factor.

\section{Methods}

\section{Study design and patients}

In this study we retrospectively analysed 180 patients with CLL exposed to treatment with ibrutinib. Patients were recruited from three independent cohorts from Italy (Rome Tor Vergata University, Rome Cattolica Sacro Cuore University and Catania Ferrarotto Hospital), between 2014 and 2019. Informed consent was obtained in accordance with the Declaration of Helsinki. The study was performed under the Institutional Review Board of the Centro di Riferimento Oncologico (IRCSS) of Aviano (approval numbers: IRB-05-2010 and IRB-05-2015).

Patients were 122 males and 58 females with a median age of 69 years (range, 36-90). According the modified Rai staging system, ${ }^{11} 134$ patients had an intermediate risk and 46 a high risk stage. All these parameters were considered at the time ibrutinib was initiated.

All patients received $420 \mathrm{mg}$ oral ibrutinib (Imbruvica; Janssen, Beerse, Belgium) once daily until progression or occurrence of unacceptable side effects. Median number of previous chemotherapy regimens were two (range, 0-4). Patients receiving first-line ibrutinib were $26(14.4 \%)$, of whom 24 of 26 (92\%) were TP53 mutated. Median follow-up was 25 months (range, 10-61). Seventy-three patients (40.6\%) discontinued ibrutinib either for progression $(n=42)$ or for adverse events $(n=31)$ (Table 1): 32 patients were subsequently treated with venetoclax (11 for toxicity [grade 3 or 4 World Health Organization] and 21 for progression of disease), five patients were treated with idelalisib, and the remaining 36 patients received other lines of chemotherapy $(n=12)$ or no therapy $(n=24)$. The clinical characteristics of patients are reported in Table 1 . The clinical assessment of patients with CLL to establish diagnosis and response to therapy were based both on the International Workshop on Chronic Lymphocytic Leukemia (iwCLL) criteria. ${ }^{12}$ The clinical impact of NOTCH1 $M$ and BAX/BCL-2 ratio on ibrutinib treatment was evaluated by measuring the kinetics of absolute lymphocyte counts (ALC), the reduction of lymphadenopathy, and the clinical outcome, as defined by ORR, PFS and OS.

\section{Chronic lymphocytic leukemia characterization}

Flow cytometry was employed for immunophenotypical CLL characterization and was performed with FACSCalibur or FACSCanto I flow cytometer. BCL-2 and BAX oncoproteins were analysed by flow cytometry in samples taken before starting ibrutinib. BAX/BCL-2 ratio was calculated dividing mean fluorescence intensity (MFI) of BAX by MFI of BCL-2 on CLL cells, as previously described. ${ }^{10}$ The threshold of positivity was set at $\geq 1.5$. Immunoglobulin heavy-chain variable region gene (IGHV) mutational status was performed by NGS, as previously described..$^{13,14}$ TP53 exons 2 to 11 mutational status and NOTCH1 exon 34 and 3' untranslated (UTR) region mutational status were analysed by NGS, as previously described. ${ }^{4,7}$ CLL samples were considered

Table 1. Patient characteristics $(n=180)$

\begin{tabular}{|c|c|}
\hline & No. of patients/Total cases (\%) \\
\hline Observation time & 2014-2019 \\
\hline Median age, y (range) & $69(36-90)$ \\
\hline Males & $122 / 180(68)$ \\
\hline $\begin{array}{l}\text { Modified Rai stage } \\
\text { Intermediate } \\
\text { High }\end{array}$ & $\begin{array}{l}134(74) \\
46(26)\end{array}$ \\
\hline $\begin{array}{c}\text { Number of previous regimens } \\
0 \\
1 \\
2 \\
3 \\
4\end{array}$ & $\begin{array}{c}26 / 180(14.5) \\
56 / 180(31.1) \\
74 / 180(41.1) \\
22 / 180(12.2) \\
2 / 180(1.1)\end{array}$ \\
\hline NOTCH1 mutation & $65 / 180(36.1)$ \\
\hline BAX/BCL-2 ratio $<1.50$ & $74 / 113(65.5)$ \\
\hline Trisomy 12 & $23 / 179(13)$ \\
\hline 11q deletion & $35 / 179(20)$ \\
\hline TP53 mutations/17p deletion & $66 / 178(37.1)$ \\
\hline $\begin{array}{l}\text { UM IGHV } \\
\text { CD38 } \geq 30 \%\end{array}$ & $\begin{array}{l}123 / 175(70.3) \\
54 / 113(47.8)\end{array}$ \\
\hline $\mathrm{CD} 49 \mathrm{~d} \geq 30 \%$ & $108 / 179(60.3)$ \\
\hline Median follow up (months) & $25(10-61)$ \\
\hline \multicolumn{2}{|l|}{ Response to ibrutinib therapy } \\
\hline $\begin{array}{l}\text { Complete response } \\
\text { Partial response } \\
\text { Partial response with lymphocytosis } \\
\text { Stable disease/No response }\end{array}$ & $\begin{array}{l}33 / 180(18.3) \\
51 / 180(28.3) \\
81 / 180(45.1) \\
15 / 180(8.3)\end{array}$ \\
\hline $\begin{array}{l}\text { Discontinuation } \\
\text { Progression } \\
\text { Toxicity }\end{array}$ & $\begin{array}{c}73 / 180(40.6) \\
42 / 180(35) \\
31 / 180(65)\end{array}$ \\
\hline Richter Syndrome & $13 / 180(7.2)$ \\
\hline Progression-free Survival at 2 years & $80 \%$ \\
\hline Overall Survival at 2 years & $84 \%$ \\
\hline Overall Survival at 4 years & $71 \%$ \\
\hline
\end{tabular}

y: years; IGHV: immunoglobulin heavy-chain variable region gene. No.: number. 
mutated for NOTCH1 i.e., NOTCH1 $M$, if exceeding a variant allele frequency (VAF) of $1 \%$. $^{15,16}$

\section{Redistribution lymphocytosis and nodal response}

The redistribution lymphocytosis was calculated as percent variation of ALC over the baseline values. Nodal response was calculated as percent reduction in sum of the product of diameter (SPD) values on the major lymph node regions over the baseline measurement, as reported previously. ${ }^{17}$

Additional details on the employed procedures and methods are reported in the Online Supplementary Materials and Methods.

\section{Results}

\section{NOTCH1 mutations and BAX/BCL-2: correlations with other biological parameters}

Sixty-five patients were NOTCH1 M (65 of 180, 36.11\%), with VAF levels $>1$ (Online Supplementary Table 2S). With regard to the distribution of VAF levels, 21 patients had VAF between $1 \%$ and $10 \%$, seven patients between $10.5 \%$ and $20 \%$ and 37 patients above $20 \%$. Fifty-six NOTCH1 M cases bore a single mutation, eight cases two mutations and one case three mutations. NOTCH1 $M$ cases were classified as follows: 45 delCT, six frameshift other than delCT (FS), 8 3'-UTR and six considering both missense (one) and nonsense (five) mutations (Online Supplementary Table S2). Seventy-four patients showed a BAX/BCL-2 ratio lower than 1.5 (74 of $113,65.5 \%)$. NOTCH1 $M$ were significantly associated with SPD ratio < 1.5: in fact, 34 of 38 NOTCH1 M patients showed BAX/BCL-2 ratio less than $1.5(P=0.0001)$. Moreover, NOTCH1 $M$ were strongly correlated with CD49d expression: 51 patients were both NOTCH1 $M$ and $\mathrm{CD} 49 \mathrm{~d} \geq 30 \%(P=0.0001)$. Furthermore, a significant corre- lation was found between a lower BAX/BCL-2 ratio and CD38 $>30 \%$ (41 of $54 ; P=0.030$ ) as well as between CD38 $>30 \%$ and NOTCH1 $M$ (27 of 38 patients; $P=0.0004$ ) (Table 2; Online Supplementary Table S3).

Trisomy 12 was confirmed to be strongly correlated with NOTCH1 M (18 of 23; $P=0.0002)$. There was only a trend towards significant association between NOTCH1 $M$ and IGHV UM status (48 of 62; $P=0.08$ ). On the other hand, IGHV UM status was correlated with lower BAX/BCL-2 ratio (58 of $81 ; P=0.030$ ). TP53 $M$ and/or del17 $p$ were found in 66 of 178 patients (37.1\%). Noteworthy, 23 of 178 patients $(13 \%)$ were simultaneously NOTCH1 and TP53 mutated. The distribution of clinical and biological prognostic factors according to NOTCH1 $\mathrm{M}$ is shown in Table 2. The distribution of prognostic factors according to the BAX/BCL-2 ratio and CD38 was obtained in 113 patients from Rome and shown in Table 2 and the Online Supplementary Table S3.

\section{Relevance of NOTCH1 mutations as biological prognostic parameter}

The mean peripheral lymphocyte percentage change from baseline, calculated at 3 months on ibrutinib, was lower in NOTCH1 M patients than in NOTCH1 wild-type (WT) patients (14\% vs. 54\%; $P=0.022$, Mann-Whitney test), thus confirming a reduced redistribution lymphocytosis (Figure 1A).

Moreover, the mean percent SPD change, calculated at 6 months on ibrutinib, was lower in NOTCH $1 M$ patients than in NOTCH1 WT patients $(53 \%$ vs. $80 \%$; $P<0.0001$, MannWhitney test), confirming a significant poor nodal response (Figure 1B). Moreover, we compared NOTCH1 M plus lower BAX/BCL-2 ratio versus NOTCH1 $M$ plus higher BAX/BCL-2 ratio with respect to redistribution lymphocy-
A

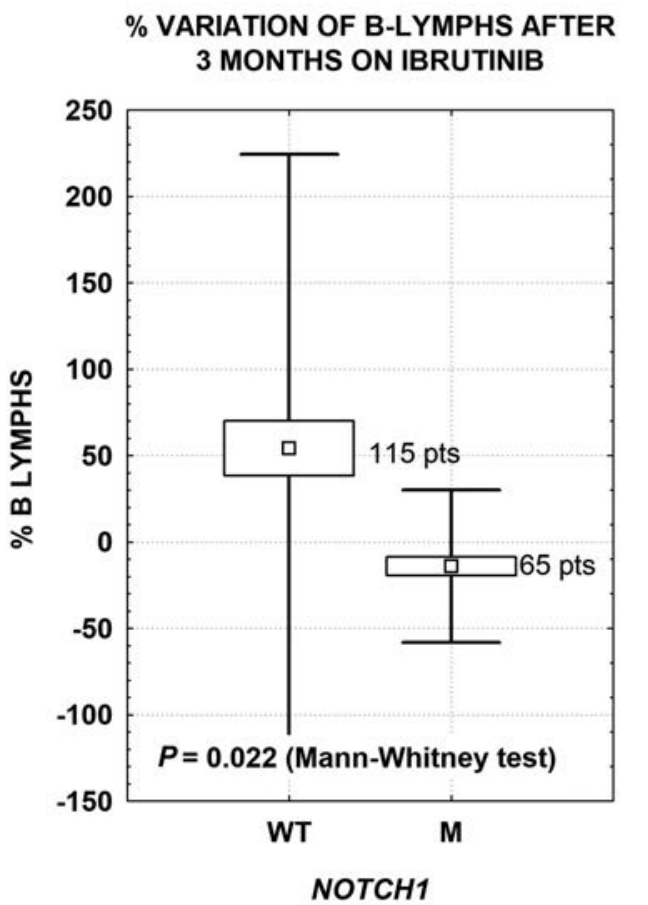

B

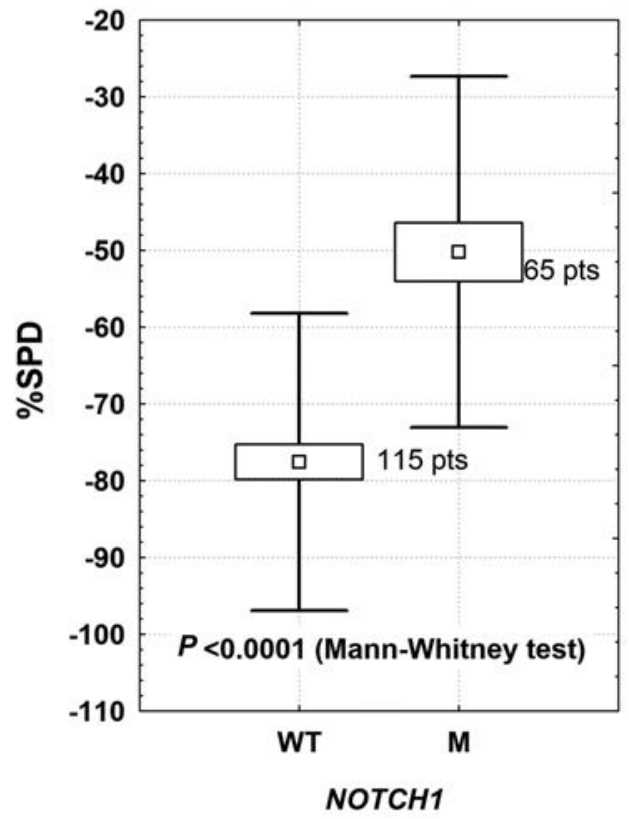

Figure 1. Box plots by NOTCH1 wild-type and mutated groups showed significant lower redistribution lymphocytosis after 3 months on ibrutinib treatment in NOTCH1 $M$ patients (A) and equally lower sum of the product of diameter (SPD) values after 6 months in NOTCH1 M patients (B). pts: points; WT: wild-type; M: mutated; B LYMPHS: B lymphocytes. 
Table 2. Distribution of prognostic factors in chronic lymphocytic leukemia according to NOTCH1 mutations

\begin{tabular}{|c|c|c|c|c|c|c|c|c|}
\hline \multirow[b]{2}{*}{ Parameter } & \multicolumn{2}{|c|}{ NOTCH1 } & \multirow[b]{2}{*}{$P \|$} & \multirow[b]{2}{*}{$n \S$} & \multirow{2}{*}{ 4-year 0S,\% } & \multirow[b]{2}{*}{ P* } & \multirow[b]{2}{*}{ 4-year PFS, \% } & \multirow[b]{2}{*}{ P* } \\
\hline & Mutated & Wild-type & & & & & & \\
\hline \multicolumn{9}{|l|}{ Age } \\
\hline$<60$ years & 31 & 54 & 0.52 & 180 & 85 & 0.29 & 81 & 0.72 \\
\hline$>60$ years & 34 & 61 & & & 95 & & 89 & \\
\hline $\begin{array}{l}\text { Sex } \\
\quad \text { Male } \\
\quad \text { Female }\end{array}$ & $\begin{array}{l}45 \\
20\end{array}$ & $\begin{array}{l}77 \\
38\end{array}$ & 0.44 & 180 & $\begin{array}{l}122 \\
58\end{array}$ & 0.71 & $\begin{array}{l}115 \\
55\end{array}$ & 0.55 \\
\hline \multicolumn{9}{|l|}{ Mod-Rai } \\
\hline Intermediate & 10 & 30 & 0.32 & 180 & 140 & 0.81 & 40 & 0.33 \\
\hline High & 55 & 85 & & & 40 & & 130 & \\
\hline $\begin{array}{l}\text { Lines of therapy } \\
\quad \leq 2 \\
>2\end{array}$ & $\begin{array}{l}55 \\
10\end{array}$ & $\begin{array}{l}101 \\
14\end{array}$ & 0.54 & 180 & $\begin{array}{l}156 \\
24\end{array}$ & 0.004 & $\begin{array}{r}148 \\
22\end{array}$ & 0.002 \\
\hline \multicolumn{9}{|l|}{ CD49d } \\
\hline$<30 \%$ & 14 & 57 & 0.0001 & 179 & 71 & 0.23 & 68 & 0.045 \\
\hline$>30 \%$ & 51 & 57 & & & 108 & & 101 & \\
\hline $\begin{array}{l}\text { CD38 } \\
<30 \% \\
>30 \%\end{array}$ & $\begin{array}{l}11 \\
27\end{array}$ & $\begin{array}{l}48 \\
27\end{array}$ & 0.0004 & 113 & $\begin{array}{l}59 \\
54\end{array}$ & 0.52 & $\begin{array}{l}56 \\
50\end{array}$ & 0.36 \\
\hline \multicolumn{9}{|l|}{ FISH } \\
\hline $\begin{array}{l}\text { Normal/del13q } \\
+12,11 q-, 17 p-\text { del11q, del17p) }\end{array}$ & $\begin{array}{l}24 \\
41\end{array}$ & $\begin{array}{l}46 \\
68\end{array}$ & 0.0002 & 179 & $\begin{array}{c}71 \\
107\end{array}$ & 0.49 & $\begin{array}{l}70 \\
98\end{array}$ & 0.46 \\
\hline $\begin{array}{l}I G H V \\
\text { Mutated } \\
\text { Unmutated }\end{array}$ & $\begin{array}{l}14 \\
48\end{array}$ & $\begin{array}{l}38 \\
75\end{array}$ & 0.080 & 175 & $\begin{array}{c}52 \\
123\end{array}$ & 0.76 & $\begin{array}{c}48 \\
117\end{array}$ & 0.036 \\
\hline \multicolumn{9}{|l|}{ TP53 } \\
\hline $\begin{array}{l}\text { Mutated } \\
\text { Wild-type }\end{array}$ & $\begin{array}{l}23 \\
40\end{array}$ & $\begin{array}{l}43 \\
72\end{array}$ & 0.52 & 178 & $\begin{array}{c}66 \\
112\end{array}$ & 0.028 & $\begin{array}{c}59 \\
109\end{array}$ & 0.022 \\
\hline $\begin{array}{l}\text { BAX/BCL-2 ratio } \\
\quad<1.5 \\
\quad>1.5\end{array}$ & $\begin{array}{c}34 \\
4\end{array}$ & $\begin{array}{l}40 \\
35\end{array}$ & 0.0001 & 113 & $\begin{array}{l}74 \\
39\end{array}$ & 0.013 & $\begin{array}{l}67 \\
39\end{array}$ & 0.0019 \\
\hline
\end{tabular}

TI Fisher exact tests were performed to evaluate the association between NOTCH1 mutations or wild-type and other prognostic factors. $\S$ Values refer to the number of cases analysed for a given feature. ${ }^{*} P$-values were calculated by the log-rank test in univariate analysis. PFS: progression-free survival; OS: overall survival; FISH: fluorescence in situ hybridisation; IGHV: immunoglobulin heavy-chain variable region gene.

tosis and lymph node shrinkage. No significant differences were found between these two subsets (Online Supplementary Figure S3).

\section{NOTCH1 mutations, BAX/BCL-2 ratio and their impact on clinical outcome}

According to clinical endpoints, ORR was $91 \%$ [complete response (CR): $18 \%$, partial response (PR): $28 \%$, PR with lymphocytosis (PR-L): 45\%] (Table 1). The estimated 2-year and 4-year OS were $84 \%$ and $71 \%$, respectively (Table 1; Online Supplementary Figure S4). Noteworthy, OS was longer in patients previously treated with one line of chemoimmunotherapy before ibrutinib $(P=0.02$, Online Supplementary Figure S5). PR and PR-L were significantly correlated with NOTCH1 M (30 of 65 and 22 of 65, respectively; $P=0.00001$, Online Supplementary Table S4). Of note, PR, PR$\mathrm{L}$ and chemoresistance were also associated with lower BAX7BCL-2 ratio (23 of 29,33 of 52 and nine of nine, respectively; $P=0.002$, Online Supplementary Table S5). Interestingly, discontinuation due to disease progression was more frequent in NOTCH1 $M$ patients than in NOTCH1 WT patients $(P=0.034$, Online Supplementary Table S4). Significant shorter PFS and OS were observed in NOTCH1 $M$ patients $(34 \%$ vs. $76 \%$ and $56 \%$ vs. $83 \%$ at 3 years, respectively; $P=0.00002$ and $P=0.001$; Figure $2 \mathrm{~A}$ and $B)$. There were no significant differences among VAF range
Table 3. Multivariate Cox regression analysis

\begin{tabular}{lcccc} 
& \multicolumn{2}{c}{ PFS } & \multicolumn{2}{c}{ OS } \\
& 168 patients & \multicolumn{1}{c}{178 patients } \\
Parameter & HR & $\boldsymbol{P}$ & HR & $\boldsymbol{P}$ \\
NOTCH1 M & 3.89 & 0.00006 & 2.64 & 0.0039 \\
$>2$ lines of therapy & 2.88 & 0.0040 & 2.43 & 0.015 \\
\hline TP53 M & 2.05 & 0.028 & 1.94 & 0.047 \\
\hline
\end{tabular}

PFS: progression-free survival, OS: overall survival: M: mutant; HR: hazard ratio.

$1-10 \%, 10.5-20 \%$ and above $20 \%$ with respect to PFS and OS, as shown in the Online Supplementary Figures S6 and S7. Moreover, we restricted the analysis of NOTCH1 to the relapse setting only (154 of 180 patients) obtaining similar significant results regarding PFS and OS (Online Supplementary Figures S8 and S9).

\section{Additive prognostic properties of NOTCH1 mutations and $B A X / B C L-2$ ratio}

In order to obtain a better refinement in the prognostic assessment of PFS and OS, we combined the values of the BAX/BCL-2 ratio with those of NOTCH1. Within the subset of 113 patients from Rome, shorter PFS and OS were detected both in patients with NOTCH1 M $(46 \%$ vs. $83 \%$ and $68 \%$ vs. $86 \%$ at 3 years, respectively; $P=0.0019$ and 
$P=0.031$, Online Supplementary Figure S10A and B) and with lower BAX/BCL-2 ratio $(60 \%$ vs. $97 \%$ and $72 \%$ vs. $94 \%$ at 3 years, respectively; $P=0.019$ and $P=0.013$, Figure $3 \mathrm{~A}$ and $B)$. Therefore, higher or lower BAX/BCL-2 ratio combined with NOTCH1 WT or NOTCH1 $M$ identified two subsets of patients, the former with the best prognosis and the latter with the worst prognosis with respect to both PFS (97\% vs. $42 \% ; P=0.0002$, Figure $4 \mathrm{~A})$ and $\mathrm{OS}(94 \%$ vs. $63 \% ; P=0.005$, Figure $4 \mathrm{~B}$ ), confirming the true additive prognostic properties of these two prognosticators.

\section{Multivariate analysis}

The clinical impact of NOTCH1 as independent prognosticator was checked by multivariate Cox proportional hazards analysis applied to models including two other prognosticators proven to be significant in univariate analysis (Table 2). With respect to PFS, NOTCH1 $M$ $(P=0.0002)$ were confirmed as an adverse independent prognostic factor $(P=0.00006)$ together with $>2$ previous lines of therapy $(P=0.004)$ and TP53 $M(P=0.028)$ (Table 3$)$. Similarly, in a multivariate analysis of OS, NOTCH1 $M$ retained an independent prognostic value $(P=0.0039)$ together with $>2$ previous lines of therapy $(p=0.015)$ and TP53 $M(\mathrm{p}=0.047)$ (Table 3$)$. NOTCH1 $M$ and $>2$ previous lines of therapy were confirmed as independent prognosticators for PFS ( $P=0.035$ and $P=0.015$, respectively) also in a model that included the BAX7BCL-2 ratio, available in a smaller subset of cases $(n=113$, Online Supplementary Table S6). Conversely, in the same subset of patients, no factor emerged as independent prognosticator for OS (Online Supplementary Table S6).

\section{Discussion}

In the present study we evaluated the efficacy of ibrutinib treatment in the high-risk NOTCH1 $M$ CLL group and correlated NOTCH1 $M$ to BAX/BCL-2 ratio, a value reflecting the susceptibility of cells to apoptosis. Efficacy of ibrutinib remained high at 4-year follow-up in almost all pre-treated patients with CLL, with $71 \%$ of patients alive and progression free, similarly to other studies. ${ }^{17}$ Moreover, ibrutinib was more effective in patients previously treated with only one line therapy, compared to patients previously treated

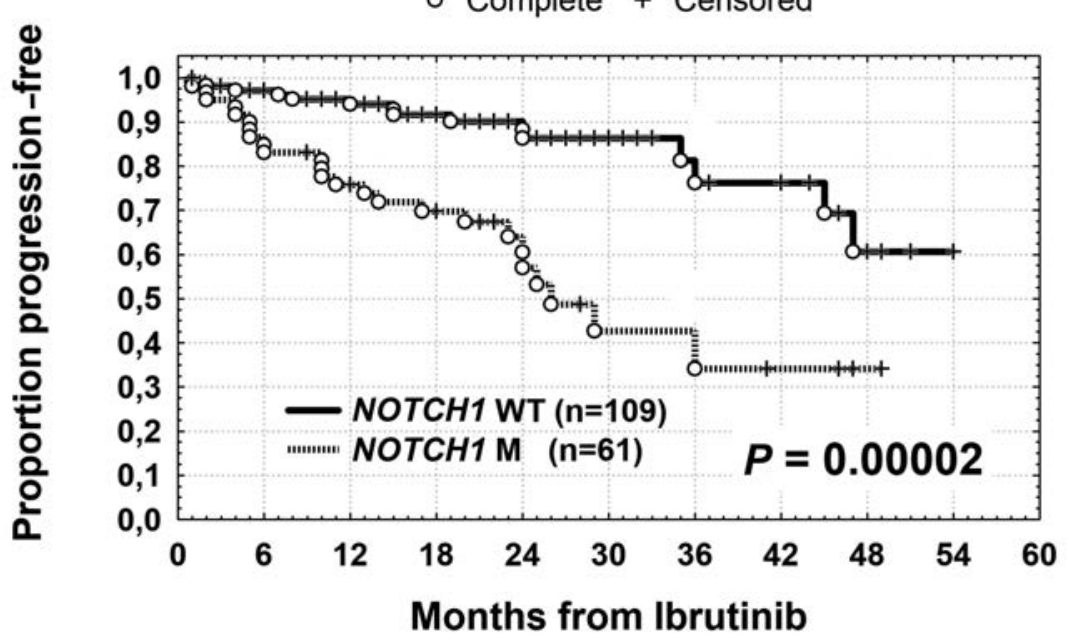

B

○ Complete + Censored

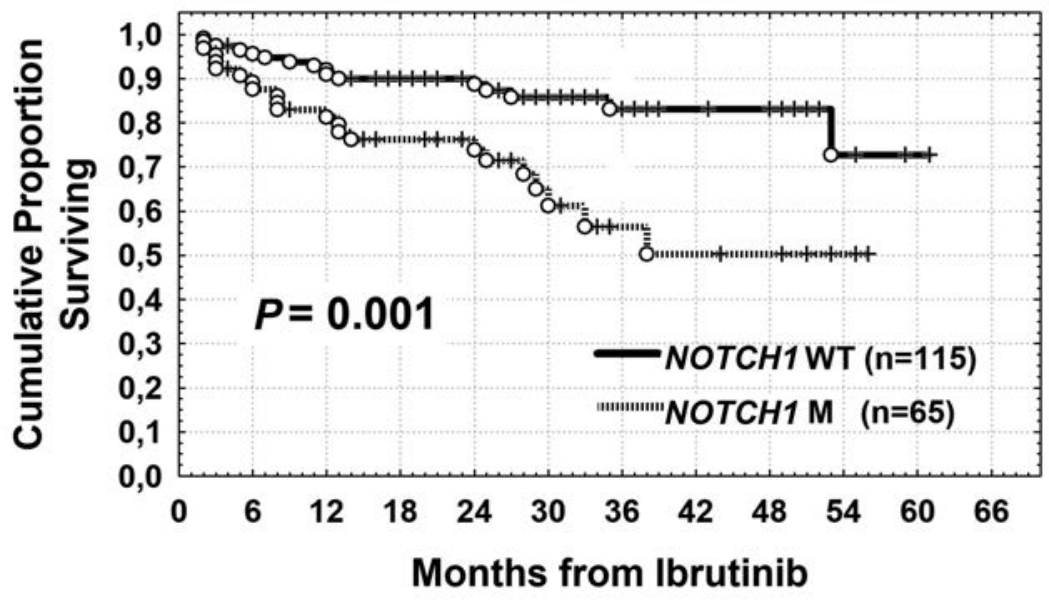

Figure 2. Progression-free survival and overall survival curves based on NOTCH1. Kaplan-Meier plot comparing progression-free survival (PFS) (A) and overall survival (OS) (B) based on NOTCH1. NOTCH1 mutated (NOTCH1 M) patients experienced both a shorter PFS and OS. 
with $>2$ lines of therapy (Online Supplementary Figure S5). On the other hand, the clinical outcome was similar for patients receiving first-line ibrutinib and patients with one previous therapy, probably due to the high incidence of TP53 mutated patients (24 of 26) in first-line setting (Online Supplementary Figure S5).

In CLL, the frequency of NOTCH1 $M$ cases between 6$12 \%$ if evaluated at presentation, increases to about $15-$ $20 \%$ in the context of fludarabine refractory patients. ${ }^{18,19}$ The higher frequency of NOTCH1 $\mathrm{M}$ characterizing our cohort of patients (36\%) could be attributed both to the previous lines of chemotherapy and to the very low cut-off $(>1 \%)$ chosen for NOTCH1 M. The adverse clinical outcome of patients with NOTCH1 M CLL was confirmed in univariate analysis in several independent cohorts of patients treated with chemo-immunotherapy ${ }^{2024}$ Since clonal CLL cells accumulate because of prolonged survival due to impairment of apoptosis, the analysis of the BAX/BCL-2 ratio could be a valid tool to provide information on the chemo-sensitivity of CLL cells. ${ }^{9,10}$

We addressed the clinical impact of both NOTCH1 M, evaluated by NGS, and BAX/BCL-2 ratio, determined by flow cytometry, in patients with CLL homogeneously treated with ibrutinib, mainly in a (R/R) setting. Determination of both parameters was done prior to starting ibrutinib therapy.

The NGS approach used for NOTCH1 $M$ analysis allowed detection of allele frequency as low as $1 \%$, highlighting the presence of subclonal mutations in $32 \%$ of total NOTCH1 $M$ cases..$^{1,16,26}$ Of note, subclonal NOTCH1 M (i.e., $\mathrm{VAF}<10 \%)$ had similar prognostic impact as clonal mutations (Online Supplementary Figures S6 and S7); consistently a receiver operating characteristic curve analysis confirmed the use of $1 \%$ as optimal cut-off (Online Supplementary Figures S2). In this context, detection of NOTCH1 M by NGS could be viewed as a useful tool for clinical follow-up of patients as well as for minimal residual disease studies, although the latter use remains speculative at the moment. From a biological point of view, we found a significant relationship between NOTCH1 and some other prognosticators. In particular, a significant correlation between NOTCH1 $M$ and higher CD49d or CD38 expressions was

A

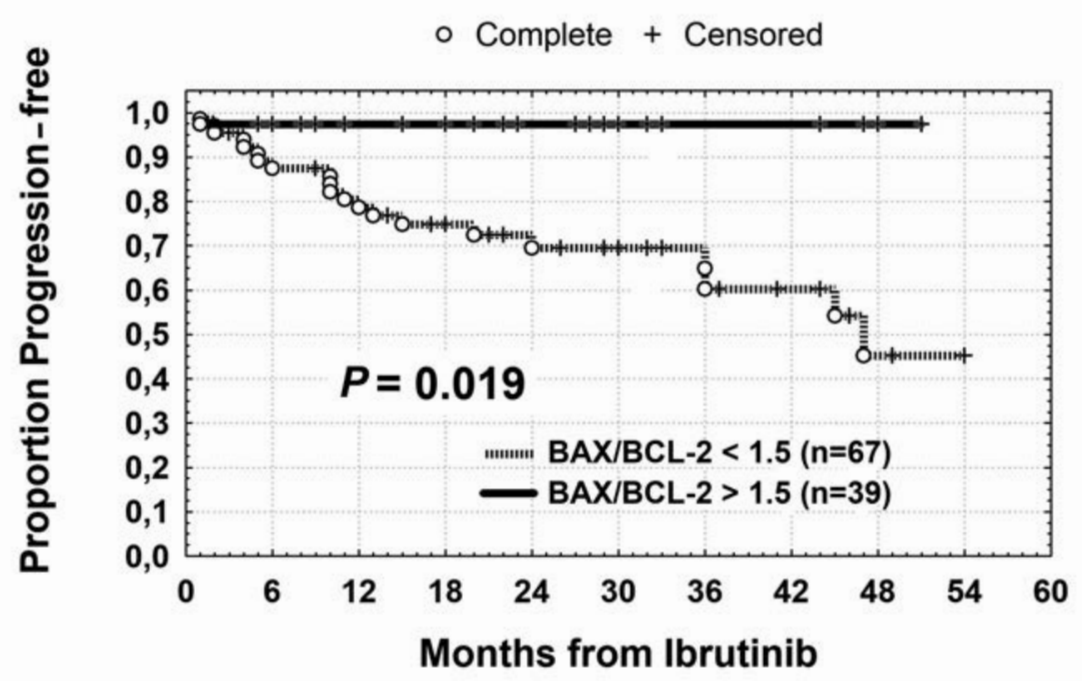

$\circ$ Complete + Censored

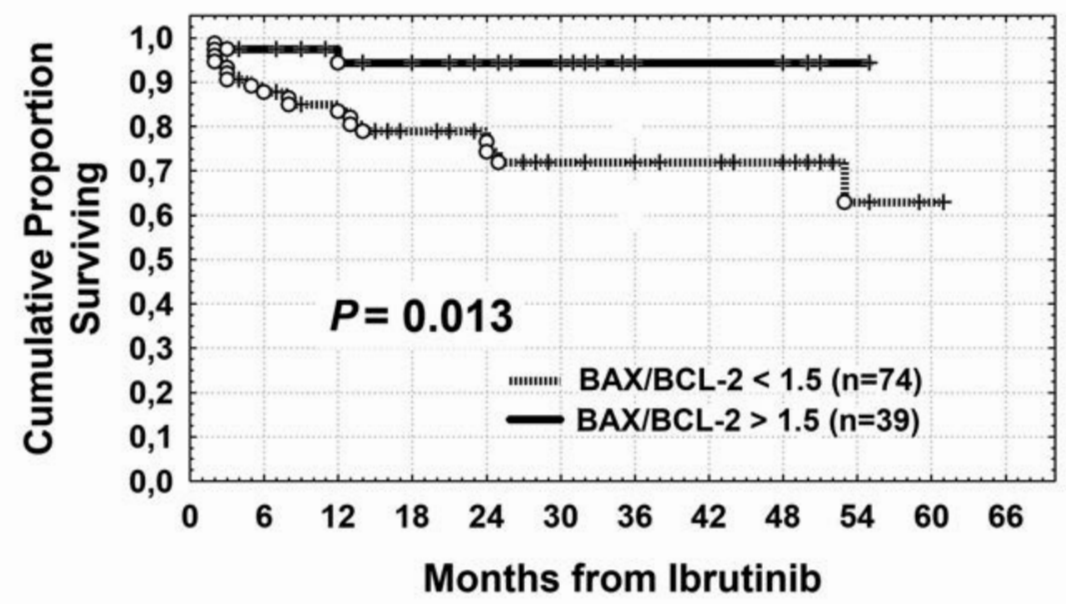

Figure 3. Progression-free survival and overall survival curves based on BAX/BCL-2 ratio within the subset of 113 patients from Rome. Kaplan-Meier plot comparing progression-free survival (PFS) (A) and overall survival (OS) (B) based on the BAX/BCL-2 ratio. Patients with a BAX/BCL-2 ratio $<1.5$ experienced both shorter PFS and OS. 
observed, as well a trend towards an association between NOTCH1 $M$ and IGHV UM status, in keeping with previous observations by us and others. ${ }^{27,21,4,28}$ Further, co-occurrence of NOTCH1 $M$ and TP53 $M$ characterized $13 \%$ of our patients (23 of 178), a rather high percentage if compared to previous reports where concomitant NOTCH1 M and TP53 M, preferentially affecting the same leukemic cells, ${ }^{29}$ accounted for $1.2-2.6 \%$ of CLL patients. ${ }^{20,23}$ This may be due to the high number of pre-treated patients and to the low cut-off chosen by us for NOTCH1 $M$ detection. We confirmed that NOTCH1 $M$ were strongly correlated with trisomy 12, in line with previous reports describing a high NOTCH1 $M$ rate in CLL cases with isolated trisomy 12 and a lower frequency in cases characterized by additional chromosomal abnormalities. ${ }^{30-32}$ In particular, a mutation frequency of $41.9 \%$ was reported in aggressive trisomy 12 cases, suggesting a pivotal role of NOTCH1 activation in this group. ${ }^{33}$ Moreover, we observed here a lower BAX/BCL-2 ratio in NOTCH1 $M$ patients, in keeping with our previous studies showing NOTCH1-dependent activation of the NF-kB pathway that may result in the upregulation of target genes, including $B C L-2 .{ }^{4}$
The strong correlation between lower BAX/BCL-2 ratio and NOTCH1 $M$ suggests that the poor prognosis of NOTCH1 $M$ patients may be related to the lack of apoptosis, although these observations need further confirmation.

The variability in the degree and kinetics of ibrutinibinduced recirculation lymphocytosis has been highlighted by several studies, ${ }^{34,35}$ and was also confirmed in the present study. Here we show that at 3 months on ibrutinib, the typical ibrutinib-induced peak of lymphocytosis is observed in NOTCH1 WT patients, but not in NOTCH1 $M$ cases. Moreover, even though the analysis of nodal response confirmed an overall significant reduction in organomegaly and lymph node size in most cases at 6 months on ibrutinib, NOTCH1 $M$ cases experienced a significant lower nodal response compared to NOTCH1 WT cases. These results may be explained by the strong correlation between CD49d overexpression and NOTCH1 M (51 of 65 cases), in line with the reported involvement of the NOTCH1 pathway in the regulation of CD49d expression. ${ }^{4}$ Consistently, CD49d associates with nodal presentation and subsequent development of lymphadenopathy in patients with CLL. ${ }^{36}$
A

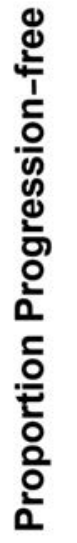

○ Complete + Censored

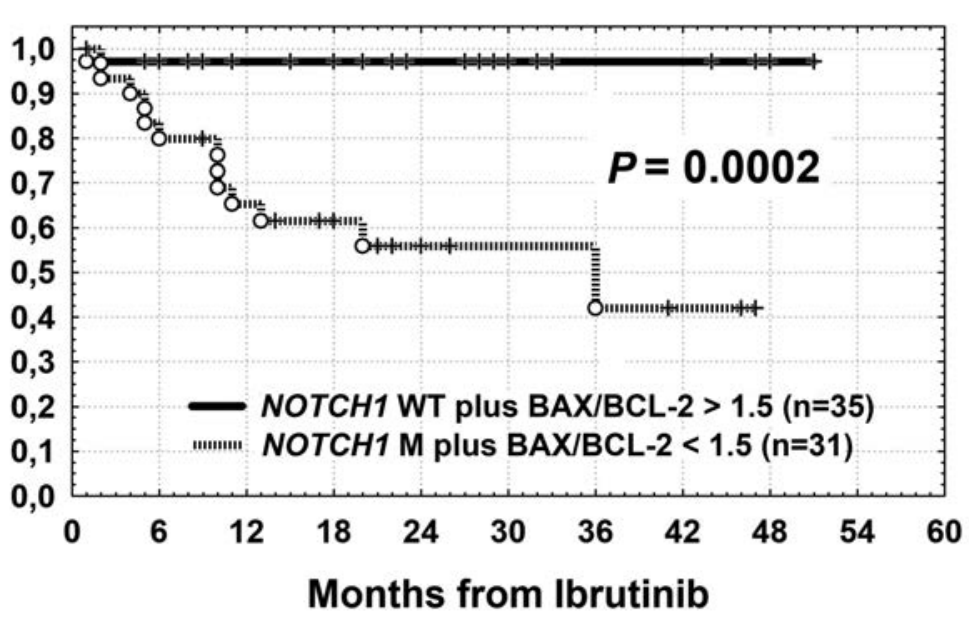

- Complete + Censored

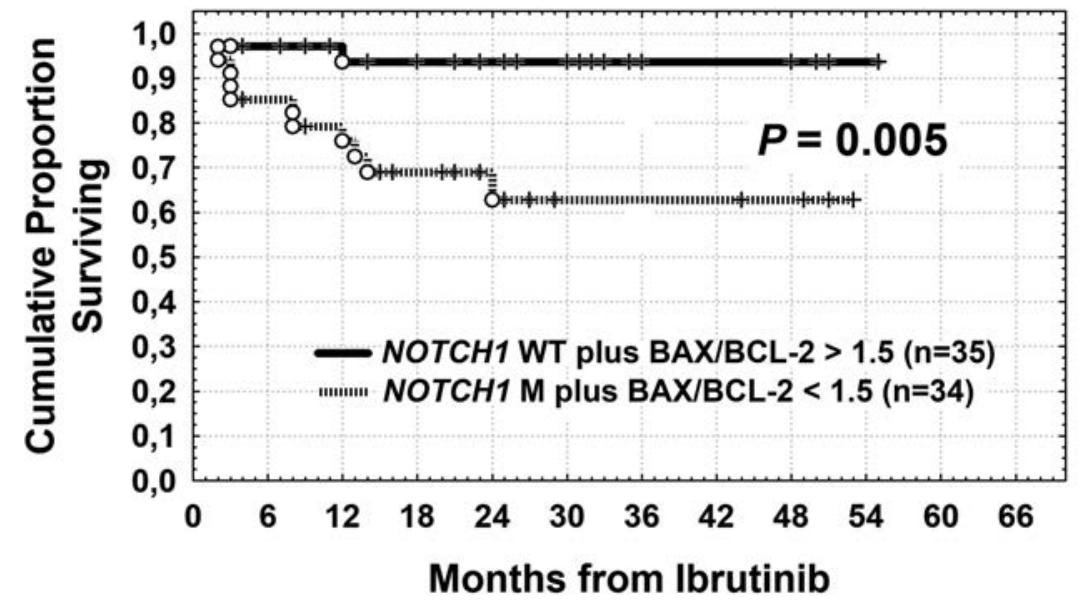

Figure 4. Progression-free survival and overall survival curves in relation to combined BAX/BCL-2 ratio and NOTCH1. Progression-free survival (PFS) and overall survival (OS) were shorter within the NOTCH1 mutated (NOTCH1 M) plus BAX/BCL-2 <1.5 subgroup (A-B), showing additive prognostic properties. 
Moreover, CD49d expression identifies cases with reduced lymphocytosis and inferior nodal response upon ibrutinib treatment, suggesting the retention of CD49d-expressing cells in tissue sites via activated VLA-4.

Consistently with the high frequency of pre-treated patients in our cohort (154 of 180), the OS values at 2 and 4 years $(84 \%$ and $71 \%$ respectively), were similar to those reported for the phase III RESONATE study in patients with previously treated CLL/SLL. ${ }^{37}$

We have recently reported that NOTCH1 $M$ identify a subgroup of patients with CLL with worse prognosis in the setting of a rituximab-based induction and consolidation treatment. ${ }^{38}$ Here, we described a negative prognostic impact of NOTCH1 $M$ also in the ibrutinib setting. Our findings differ from those resulting from the extended follow up from the RESONATE study of relapsed/refractory CLL, where the presence of NOTCH1 $M$ did not negatively affect the efficacy of ibrutinib on disease progression outcomes. $^{37}$ This difference can be explained by the very low cut-off $(>1 \%)$ chosen for NOTCH1 $M$ in our study, although for the validation of these findings additional independent cohorts are needed.

The here reported capacity of BAX/BCL-2 index to identify patients with a different response to ibrutinib could be of interest in the light of the treatments protocols associating $\mathrm{B}$-cell receptor inhibitors and $\mathrm{BH} 3$ mimetics such as venetoclax. ${ }^{39}$

Moreover, the additive negative prognostic value of NOTCH1 $M$ and low BAX/BCL-2 ratio described by us, further support the rationale to improve the efficacy of ibrutinib by using the BCL-2 inhibitor venetoclax in patients with NOTCH1 mutated CLL. ${ }^{10}$ Interestingly, an additive prognostic impact of the combination of BAX/BCL-2 and NOTCH1 $M$ in the setting of chemo-immunotherapy was also reported by us. ${ }^{10}$

Several independent cohorts of patients confirmed the adverse clinical outcome of NOTCH1 $M$ with CLL in univariate analysis, ${ }^{20-23,40}$ although conflicting results are reported about its independent prognostic effect. In particular, NOTCH1 $M$ did not retain independent significance as a predictor of time-to-first treatment in one of the largest series of patients with CLL, ${ }^{41}$ while in another study it emerged as an independent predictor of shorter survival, along with TP53 abnormalities. ${ }^{42}$ Here NOTCH1 $M$ were confirmed to be an independent prognostic factor together with previous lines of therapy and TP53 both with respect to PFS and OS. The apparent higher prognostic impact of NOTCH1 $M$ compared to TP53 mutation, as emerged in our multivariable analysis, may be explained by the greater number of TP53 mutated cases treated first line with ibrutinib, hence with a better prognosis than NOTCH1 $M$ cases that were more frequently treated with ibrutinib in second or further lines of therapy.
The current use of B-cell receptor and BCL-2 inhibitors led to high-rate improvement of outcome in CLL. However, several issues remain, resulting in resistance/progression thus limiting the eradication of the tumour. The growing evidence for a critical role of the NOTCH1 pathway in CLL makes this cancer gene a target to design tailored treatments for this peculiar subset through specific NOTCH1targeted therapies. In this context, $\gamma$-secretase inhibitors are the most extensively explored anti-NOTCH1 molecules and their combination with fludarabine demonstrated antitumour effects in primary CLL with NOTCH1 $M{ }^{43}$ Noteworthy, a humanized antibody targeting NOTCH1 (clinicaltrials gov. Identifier: OMP-52M51) entered phase I trial in relapsed/refractory lymphoid malignancies. ${ }^{44}$ However, to date, the future treatment of CLL with NOTCH1 $M$ relies on the association of small molecule inhibitors targeting both the BCR pathway and the antiapoptotic BCL-2 protein.

\section{Disclosures}

No conflicts of interest to disclose.

\section{Contributions}

GDP and VG designed the study, interpreted data, performed statistical analysis, wrote the manuscript and gave final approval of the manuscript; $A B$ and $A Z$ contributed to study design and data interpretation and to write the manuscript; $L L, A C$ and $M I D P$ contributed to interpret the data and to write the manuscript; AZ, FMR and GDP obtained flow cytometric data; FMR performed FISH cytogenetic analysis; VG, FP and RB investigated IGHV, NOTCH1 and TP53 mutations; FB, SA, GG, AV contributed to study design and data interpretation; II, MP, PdF, $M C$ recruited the patients and collected clinical data.

\section{Acknowledgments}

This study was supported in part by Ministero dell'Università e della Ricerca Scientifica e Tecnologica (MURST), Programmi di Ricerca di Interesse Nazionale; Ministero della Salute (Ricerca Finalizzata Istituto di Ricovero e Cura a Carattere Scientifico [IRCCS], Rome, Italy; Associazione Italiana Ricerca Cancro (AIRC), Investigator Grant IG-21687 (to V.G.); Progetto Ricerca Finalizzata PE 2016-02362756, Ministero della Salute, Rome, Italy (to V.G.); Progetto Ricerca Finalizzata RF-2018-12365790 (to A.Z.); Fondazione Cariplo (grant 2012-0689); Associazione Italiana contro le Leucemie, Linfomi e Mielomi (AIL), Venezia Section, Pramaggiore Group, Italy; Fondazione per la Vita di Pordenone, Italy; Ricerca Scientifica Applicata, Regione Friuli Venezia Giulia ("Linfonet" Project), Trieste, Italy; "5×1000 Intramural Program", Centro di Riferimento Oncologico, Aviano, Italy.

The authors thank the members of their Departments of Hematology clinical staff for their invaluable support to this CLL clinical research program.

\section{References}

1. Nadeu F, Delgado J, Royo C, et al. Clinical impact of clonal and subclonal TP53, SF3B1, BIRC3, NOTCH1, and ATM mutations in chronic lymphocytic leukemia. Blood. 2016;127(17):2122-2130.

2. Puente XS, Beà S, Valdés-Mas R, et al. Noncoding recurrent mutations in chronic lymphocytic leukaemia. Nature. 2015;526 (7574):519-524.
3. Rossi D, Spina V, Bomben R, et al Association between molecular lesions and specific B-cell receptor subsets in chronic lymphocytic leukemia. Blood. 2013;121(24):4902-4905.

4. Benedetti D, Tissino E, Pozzo F, et al NOTCH1 mutations are associated with high CD49d expression in chronic lymphocytic leukemia: link between the NOTCH1 and the NF- $\mathrm{BB}$ pathways. Leukemia. 2018;32(3):654-662.
5. Stilgenbauer S, Schnaiter A, Paschka P, et al Gene mutations and treatment outcome in chronic lymphocytic leukemia: results from the CLL8 trial. Blood. 2014;123(21):32473254

6. Roberts AW, Ma S, Kipps TJ, et al. Efficacy of venetoclax in relapsed chronic lymphocytic leukemia is influenced by disease and response variables. Blood. 2019;134(2):111122.

7. Tissino E, Benedetti D, Herman SEM, et al. 
Functional and clinical relevance of VLA-4 (CD49d/CD29) in ibrutinib-treated chronic lymphocytic leukemia. J Exp Med. 2018;215 (2):681-697.

8. Pepper C, Hoy T, Bentley P. Elevated Bcl2/Bax are a consistent feature of apoptosis resistance in B-cell chronic lymphocytic leukaemia and are correlated with in vivo chemoresistance. Leuk Lymphoma. 1998;28 (3-4):355-361.

9. Williamson KE, Kelly JD, Hamilton PW, et al. Bcl-2/Bax ratios in chronic lymphocytic leukaemia and their correlation with in vitro apoptosis and clinical resistance. Br J Cancer. 1998;78(4):553-554

10. Del Principe MI, Dal Bo M, Bittolo T, et al. Clinical significance of bax/bcl-2 ratio in chronic lymphocytic leukemia. Haematologica. 2016;101(1):77-85.

11. Rai KR, Han T. Prognostic factors and clinical staging in chronic lymphocytic leukemia. Hematol Oncol Clin North Am. 1990;4(2):447-456.

12. Hallek M, Cheson BD, Catovsky D, et al. iwCLL guidelines for diagnosis, indications for treatment, response assessment, and supportive management of CLL. Blood. 2018;131(25):2745-2760.

13. Degan M, Bomben R, Dal Bo $M$, et al. Analysis of $\mathrm{IgV}$ gene mutations in $\mathrm{B}$ cell chronic lymphocytic leukaemia according to antigen-driven selection identifies subgroups with different prognosis and usage of the canonical somatic hypermutation machinery. Br J Haematol. 2004;126(1):2942 .

14. Bomben R, Dal Bo M, Zucchetto A, et al. Mutational status of $\operatorname{IgV}(\mathrm{H})$ genes in B-cell chronic lymphocytic leukemia and prognosis: percent mutations or antigen-driven selection? Leukemia. 2005;19(8):1490-1492.

15. Thorvaldsdóttir H, Robinson JT, Mesirov IP. Integrative Genomics Viewer (IGV): highperformance genomics data visualization and exploration. Brief Bioinform. 2013;14 (2):178-192.

16. D'Agaro T, Bittolo T, Bravin V, et al. NOTCH1 mutational status in chronic lymphocytic leukaemia: clinical relevance of subclonal mutations and mutation types. $\mathrm{Br}$ J Haematol. 2018;182(4):597-602.

17. Byrd JC, O'Brien S, James DF. Ibrutinib in relapsed chronic lymphocytic leukemia. N Engl J Med. 2013;369(13):1278-1279.

18. Lionetti M, Fabris S, Cutrona G, et al. Highthroughput sequencing for the identification of NOTCH1 mutations in early stage chronic lymphocytic leukaemia: biological and clinical implications. $\mathrm{Br} \mathrm{J}$ Haematol. 2014;165(5):629-639.

19. Fabbri G, Rasi S, Rossi D, et al. Analysis of the chronic lymphocytic leukemia coding genome: role of NOTCH1 mutational activation. J Exp Med. 2011;208(7):1389-1401.

20. Weissmann S, Roller A, Jeromin S, et al.
Prognostic impact and landscape of NOTCH1 mutations in chronic lymphocytic leukemia (CLL): a study on 852 patients. Leukemia. 2013;27(12):2393-2396.

21. Villamor N, Conde L, Martínez-Trillos A, et al. NOTCH1 mutations identify a genetic subgroup of chronic lymphocytic leukemia patients with high risk of transformation and poor outcome. Leukemia. 2013;27(5): 1100-1106.

22. Sportoletti P, Baldoni S, Del Papa B, et al. A revised NOTCH1 mutation frequency still impacts survival while the allele burden predicts early progression in chronic lymphocytic leukemia. Leukemia. 2014;28(2):436439.

23. Del Poeta G, Dal Bo M, Del Principe MI, et al. Clinical significance of c.7544-7545 delCT NOTCH1 mutation in chronic lymphocytic leukaemia. $\mathrm{Br} \mathrm{J}$ Haematol. 2013;160(3):415-418.

24. Oscier DG, Rose-Zerilli MJ, Winkelmann N, et al. The clinical significance of NOTCH1 and SF3B1 mutations in the UK LRF CLL4 trial. Blood. 2013;121(3):468-475.

25. Chiorazzi N, Rai KR, Ferrarini M Chroniclymphocyticleukemia. N Engl J Med. 2005;352(8):804-815

26. Pozzo F, Bittolo T, Arruga F, et al. NOTCH1 mutations associate with low CD20 level in chronic lymphocytic leukemia: evidence for a NOTCH1 mutation-driven epigenetic dysregulation. Leukemia. 2016;30(1):182-189.

27. Chiaretti S, Marinelli M, Del Giudice I, et al. NOTCH1, SF3B1, BIRC3 and TP53 mutations in patients with chronic lymphocytic leukemia undergoing first-line treatment: correlation with biological parameters and response to treatment. Leuk Lymphoma. 2014;55(12):2785-2792.

28. Larrayoz M, Rose-Zerilli MJ, Kadalayil L, et al. Non-coding NOTCH1 mutations in chronic lymphocytic leukemia; their clinical impact in the UK CLL4 trial. Leukemia. 2017;31(2):510-514

29. Kantorova B, Malcikova J, Brazdilova K, et al. Single cell analysis revealed a coexistence of NOTCH1 and TP53 mutations within the same cancer cells in chronic lymphocytic leukaemia patients. Br J Haematol. 2017;178(6):979-982.

30. Del Giudice I, Rossi D, Chiaretti S, et al. NOTCH1 mutations in +12 chronic lymphocytic leukemia (CLL) confer an unfavorable prognosis, induce a distinctive transcriptional profiling and refine the intermediate prognosis of +12 CLL. Haematologica. 2012;97(3):437-441

31. Bulian P, Bomben R, Dal Bo $M$, et al. Mutational status of IGHV is the most reliable prognostic marker in trisomy 12 chronic lymphocytic leukemia. Haematologica. 2017;102(11):e443-e446.

32. López C, Delgado J, Costa D, et al. Different distribution of NOTCH1 mutations in chronic lymphocytic leukemia with isolated trisomy 12 or associated with other chromosomal alterations. Genes Chromosomes Cancer. 2012;51(9):881-889.

33. Balatti V, Lerner S, Rizzotto L, et al.Trisomy 12 CLLs progress through NOTCH1 mutations. Leukemia. 2013;27(3):740-743.

34. Herman SE, Niemann CU, Farooqui M, et al. Ibrutinib-induced lymphocytosis in patients with chronic lymphocytic leukemia: correlative analyses from a phase II study. Leukemia. 2014;28(11):2188-2196.

35. Farooqui MZ, Valdez J, Martyr S, et al Ibrutinib for previously untreated and relapsed or refractory chronic lymphocytic leukaemia with TP53 aberrations: a phase 2 , single-arm trial. Lancet Oncol. 2015;16(2): 169-176.

36. Strati P, Parikh SA, Chaffee KG, et al. CD49d associates with nodal presentation and subsequent development of lymphadenopathy in patients with chronic lymphocytic leukaemia. Br J Haematol. 2017;178(1):99105.

37. Brown JR, Hillmen P, O'Brien S, et al. Extended follow-up and impact of high-risk prognostic factors from the phase 3 RES ONATE study in patients with previously treated CLL/SLL. Leukemia. 2018;32(1):8391.

38. Dal Bo M, Del Principe MI, Pozzo F, et al. NOTCH1 mutations identify a chronic lymphocytic leukemia patient subset with worse prognosis in the setting of a rituximab-based induction and consolidation treatment. Ann Hematol. 2014;93(10):17651774

39. Del Poeta G, Del Principe M, Postorino M, et al. Apoptosis resistance and NOTCH1 mutations impair clinical outcome in chronic lymphocytic leukemia (CLL) patients treated with ibrutinib. Blood. 2017;130 (Suppl 1):261

40. Guièze R, Robbe P, Clifford R, et al. Presence of multiple recurrent mutations confers poor trial outcome of relapsed/refractory CLL. Blood. 2015;126(18):2110-2117.

41. Baliakas P, Hadzidimitriou A, Sutton LA, et al. Recurrent mutations refine prognosis in chronic lymphocytic leukemia. Leukemia. 2015:29(2):329-336

42. Rossi D, Rasi S, Fabbri G, et al. Mutations of NOTCH1 are an independent predictor of survival in chronic lymphocytic leukemia. Blood. 2012;119(2):521-529.

43. López-Guerra M, Xargay-Torrent S, Rosich $\mathrm{L}$, et al. The $\gamma$-secretase inhibitor PF03084014 combined with fludarabine antagonizes migration, invasion and angiogenesis in NOTCH1-mutated CLL cells. Leukemia. 2015;29(1):96-106.

44. Wu Y, Cain-Hom C, Choy L, et al Therapeutic antibody targeting of individual Notch receptors. Nature. 2010;464(7291): 1052-1057. 